\title{
Regionalization by fuzzy expert system based approach optimized by genetic algorithm.
}

\begin{abstract}
In recent years soft computing methods are being increasingly used to model complex hydrologic processes. These methods can simulate the real life processes without prior knowledge of the exact relationship between their components. The principal aim of this paper is perform hydrological regionalization based on soft computing concepts in the southern strip of the Caspian Sea basin, north of Iran. The basin with an area of 42,400 sq. $\mathrm{km}$ has been affected by severe floods in recent years that caused damages to human life and properties. Although some 61 hydrometric stations and 31 weather stations with 44 years of observed data (1961-2005) are operated in the study area, previous flood studies in this region have been hampered by insufficient and/or reliable observed rainfall-runoff records. In order to investigate the homogeneity (h) of catchments and overcome incompatibility that may occur on boundaries of cluster groups, a fuzzy expert system (FES) approach is used which incorporates physical and climatic characteristics, as well as flood seasonality and geographic location. Genetic algorithm (GA) was employed to adjust parameters of FES and optimize the system. In order to achieve the objective, a MATLAB programming code was developed which considers the heterogeneity criteria of less than $1(\mathrm{H}<1)$ as the satisfying criteria. The adopted approach was found superior to the conventional hydrologic regionalization methods in the region because it employs greater number of homogeneity parameters and produces lower values of heterogeneity criteria.
\end{abstract}

Keyword: Soft computing; Regionalization; Hydrological homogeneity; Fuzzy expert system; Genetic algorithm. 